\title{
PRUEBAS COMPLEMENTARIAS EN EL DIAGNÓSTICO DE LA DISFUNCIÓN ERÉCTIL: ¿CUÁLES Y CUÁNDO?
}

\author{
Venancio Chantada Abal' y Emilio Julve Villalta ${ }^{2}$.
}

'Servicio de Urología. Complejo Hospitalario Universitario A Coruña. A Coruña. España.

${ }^{2}$ Servicio de Urología. Hospital Carlos Haya. Málaga. España.

Resumen.- La introducción de los inhibidores de la PDE5 ha ampliado el ámbito de la atencion de la disfunción eréctil (DE), que ahora incluye a los médicos de atención primaria, a los urólogos, endocrinólogos, neurólogos, angiocardiólogos, psiquiatras, psicólogos y a otros especialistas. Una verdadera estrategia multidisciplinar es beneficiosa para evaluar y tratar al paciente. Para hacer un diagnóstico correcto, conviene saber que existen muchas afecciones que se asocian a la DE.

CORRESPONDENCIA

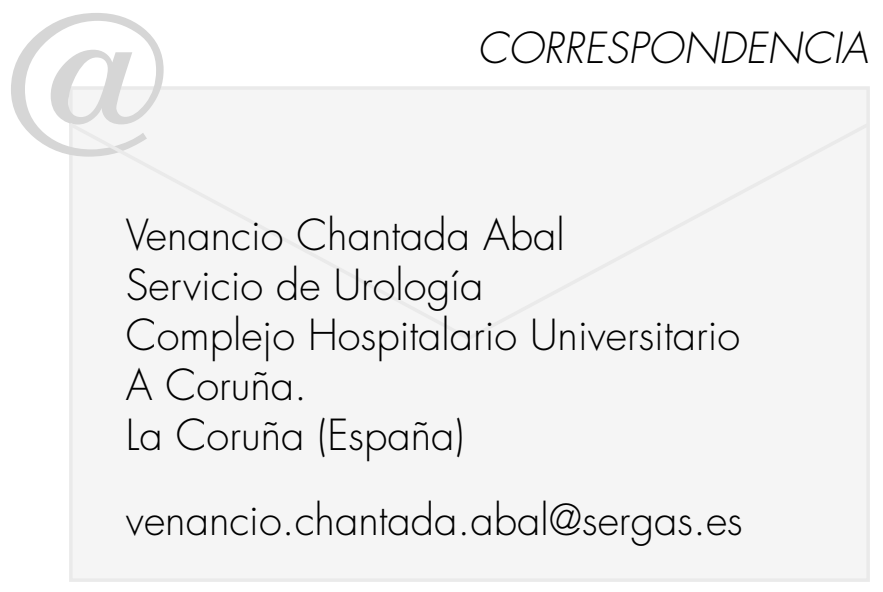

Para evaluar esta DE hay que identificar los problemas sexuales y utilizar ciertos medios, como cuestionarios adecuados y escalas de síntomas, una historia clínica y sexual detallada, la exploración física y unas pruebas analíticas elementales. En la mayoría de los casos de $D E$, no es necesario recurrir a pruebas cruentas ni derivar al paciente a un especialista, pero el médico debe saber que hay casos que exigen esa derivación y que, a veces, se necesitan mas estudios y evaluaciones

Palabras clave: Disfunción eréctil. Pruebas complementarias. Diagnóstico.

Summary.- The introduction of PDE 5 inhibitors has widen the ambit of health care in erectile dysfunction (ED), which now includes family doctors, urologists, endocrinologists, neurologists, angio-cardiologists, psychiatrists, psychologists and other specialists. To make a proper diagnosis, it is convenient to know there are many diseases associated with ED. To evaluate ED we have to identify sexual problems and to use certain means, such as adequate questionnaires and symptom scores, a detailed sexual and clinical history, physical exam and elementary lab tests. In most cases of ED it is not necessary to perform invasive tests or refer the patient to a specialist, but the doctor needs to know there are cases that require such reference, and sometimes more studies and tests are required.

Keywords: Erectile dysfunction. Complementary tests. Diagnosis. 


\section{INTRODUCCIÓN}

La disfunción eréctil (DE) es un proceso multifactorial que ha despertado gran interés entre los pacientes y sus parejas sexuales, en la comunidad médica, la industria famacéutica, la cultura popular y la sociedad en general. Desde la aparición de un tratamiento oral eficaz y exento de riesgos, como son los inhibidores selectivos de la fosfodiesterasa tipo 5 (iPDE5), el paciente y el médico han prestado más atención a la evaluación y al tratamiento de este trastorno.

La finalidad de la evaluación especializada es precisar la causa de la DE y aconsejar un tratamiento eficaz. En la mayoría de los casos es posible identificar la etiología mas probable de la DE reuniendo los datos que aporta una historia clínica detallada, la exploración física general, el examen genitourinario, y una revisión completa de las comorbilidades y de los medicamentos mas utilizados; con esto, se podrá formular un plan terapéutico.

\section{Clasificación de exploraciones complementarias en DE}

Se dispone de muchas pruebas y evaluaciones para el diagnóstico de la $\mathrm{DE}$, principalmente la historia clínica, la historia sexual y psico-social, la exploración física y las exploraciones complementarias. Dentro de este último apartado, las exploraciones empleadas en la evaluación del paciente con disfunción eréctil pueden dividirse en:

- Pruebas altamente recomendadas: Pruebas que deben realizarse en todos los pacientes y que se basan fundamentalmente en una buena exploración física que incluya tensión arterial, estado de pulsos periféricos, caracteres sexuales secundarios, sensibilidad perineal, tono del esfínter anal, morfología y morfometría peneana, así como presencia de placas de fibrosis plástica y exploración testicular y prostática.

- Pruebas recomendadas: Pruebas de valor reconocido para el estudio de la DE en la mayoría de los pacientes. Su empleo se recomienda especialmente en la evaluación inicial y se incluyen glucemia y perfil lipídico, hemoglobina glicosilada en diabéticos y evaluación del eje hipotálamo-hipofisario mediante testosterona total y libre.

- Pruebas opcionales: Pruebas útiles en la valoración de determinados tipos de casos, que ayudarán a formar un juicio clínico al médico, en el ámbito de la medicina general, entre las que se encuentran una consulta psicológica $\mathrm{y} / \mathrm{o}$ psiquiátrica, valora- ción de los niveles de prolactina, hormonas luteo y folículoestimulante (LH y FSH), hemograma y análisis sistemático de orina y antígeno prostático específico (PSA).

- Pruebas especializadas: Pruebas realizadas solo en casos determinados, dentro de una consulta especializada.

\section{Exploraciones Complementarias Especializadas en DE}

Los adelantos en el conocimiento de la fisiología de la erección y el desarrollo de la tecnología han mejorado la capacidad de definir los diferentes tipos de disfunción eréctil (neurógena, psicógena y vasculogénica). El objetivo de la evaluación especializada es definir la causa de la disfunción eréctil. En general, en Medicina se necesita un diagnóstico específico para decidir un tratamiento. En términos generales se puede afirmar que en la mayoría de los casos de disfunción eréctil no es necesario realizar exploraciones extensas ni invasivas.

Las indicaciones aceptadas para una evaluación especializada de la DE son el fracaso del tratamiento inicial, la enfermedad de Peyronie, la disfunción eréctil primaria, los antecedentes de traumatismo pélvico / perineal, los casos que requieren una intervención vascular o neuroquirúrgica, la endocrinopatía complicada, los trastornos psiquiátricos complicados, los problemas de relación complejos y los problemas médico-legales (1).

\section{Pruebas de laboratorio}

Las determinaciones analíticas sirven para detectar de forma selectiva y rentable las afecciones generales que suelen acompañar a una DE. Si analizamos las guías de la European Association of Urology, del año 2009, nos recomiendan que todos los pacientes debieran realizar una determinación sanguínea de glucosa y lípidos si no fueron analizados en los 12 meses previos. Los test hormonales deben incluir una determinación matutina de testosterona total. La medición de testosterona libre calculada es preferible a la de la testosterona total porque determina con más exactitud el hipogonadismo (2). Se pueden considerar determinaciones adicionales en pacientes seleccionados como es el PSA en los casos que se desee detectar un cáncer de próstata. Otras determinaciones hormonales como la hormona folículo-estimulante (FSH), hormona luteinizante (LH), pueden ser determinadas cuando se detecta un bajo nivel de testosterona (3). 
Los trastornos de la función endocrina afectan al comportamiento sexual, al deseo sexual y al interés por el sexo (4). En cuanto a los andrógenos se sigue debatiendo su papel en la función eréctil normal y anormal. En los varones con intenso hipogonadismo, el tratamiento sustitutivo con andrógenos mejora los resultados del registro de la tumescencia peneana nocturna, así como las erecciones evocadas por los estímulos eróticos (5). Aunque tradicionalmente se ha considerado que el papel de los andrógenos en la función eréctil normal era permisivo, cada vez existen mas pruebas de que los andrógenos son fundamentales, no sólo para el comportamiento sexual, sino también para la actividad de varias vías fisiológicas y de señalización que regulan la erección (6). En definitiva, el hipogonadismo puede afectar a la función de los agentes erectogénicos a la hora de buscar el restablecimiento completo de la función eréctil. Shabisgh el al. (7) han mostrado que la administración de testosterona a un hombre hipogonádico con una respuesta subóptima a los inhibidores de la PDE5, es altamente beneficiosa, en términos de mejoría de la respuesta. De este modo, un nivel sérico matutino de testosterona total y/o libre debe ser indicado como un test básico en un hombre con DE (8).

\section{Exploraciones Complementarias en DE: Valoración vascular}

Las exploraciones vasculares están dirigidas al diagnóstico de la disfunción eréctil de origen arterial y veno-oclusivo. En la práctica, la $\mathrm{DE}$ de origen vascular se divide en: insuficiencia arterial, venooclusiva y mixta (9). Se ha establecido una relación directa entre la calidad del aporte de sangre arterial al pene y las alteraciones vasculares más frecuentes, entre ellas el envejecimiento, la diabetes, la hipertensión arterial, las enfermedades vasculares arterioscleróticas, la hiperlipidemia y el tabaquismo (7). Las pruebas utilizadas en estos casos se clasifican dentro de tres líneas: evaluación de primera línea mediante inyección intracavernosa de prostaglandina El (PGE 1) con estimulación combinadas, evaluación de segunda línea con ecografía doppler de pene y evaluación de tercera línea mediante cavernosometría y cavernosografía de infusión dinámica y arteriografía selectiva de pene (1).

\section{Inyección intracavernosa de PGE 1 y estimulación combinadas}

Esta prueba, de fácil ejecución, consiste en la inyección intracavernosa de un vasodilatador, generalmente PGE 1, a dosis de 10 a $20 \mu \mathrm{g}$, la estimulación sexual genital o audiovisual y la evalua- ción de la erección por un observador. Después de la inyección, se realiza una evaluación subjetiva, en la que el paciente compara visualmente la erección lograda con la más perfecta que haya conseguido en su casa. Esta prueba es rápida y fácil de realizar en la consulta, y mínimamente invasiva. Es el procedimiento diagnóstico más realizado en $D E$ y permite evaluar el estado vascular del pene de forma directa y objetiva. La comparación con otras pruebas hemodinámicas indica que una respuesta normal a la inyección intracavernosa se asocia con una oclusión venosa normal. Un resultado normal en un paciente neurológicamente normal posiblemente sugiere una DE psicógena.

De acuerdo con Rosen y cols (1): "después de comparar la inyección intracavernosa con otras pruebas hemodinámicas, cuando aquella es normal, indica que la función veno-oclusiva es normal (flujo sanguíneo necesario para mantener la rigidez en valores de 0.5-3 $\mathrm{ml} / \mathrm{min}^{\prime}$. Sin embargo, si la respuesta es anormal, resulta imposible distinguir la insuficiencia arterial de la disfunción veno-oclusiva. Además, tampoco se puede saber si el estímulo farmacológico fue suficientemente intenso o si hubiera sido necesario añadir estímulos eróticos, visuales o de otra clase, para reproducir una situación de la vida real. Pueden darse falsos negativos hasta en el $20 \%$ de los casos por una irrigación arterial en el límite, y falsos positivos por ansiedad del paciente, fobia a las agujas o dosificación insuficiente. En definitiva, una prueba normal es útil para que el médico busque preferentemente las causas psicógenas de la $\mathrm{DE}$, una prueba anormal plantea varias interrogantes diagnósticas que van a necesitar más investigaciones. Por tanto, se acepta que la inyección intracavernosa es una prueba diagnóstica engañosa si se utiliza en exclusiva para excluir una DE vascular, salvo que se realice con ecografía doppler peneana simultánea (10).

\section{Ecografía doppler de pene}

Cuando está indicada la evaluación vascular, la prueba diagnóstica más informativa es la inyección intracavernosa de PGEl asociada con ecografía doppler color. Esto puede ser todo lo necesario para definir y determinar la gravedad. Esta exploración debe ser utilizada antes de considerar otras pruebas porque es el método menos invasivo para evaluar la disfunción eréctil de origen vascular, distinguir el priapismo de alto y bajo flujo y evaluar la placa de Peyronie. Aunque habitualmente se usa la inyección de PGE 1, el empleo combinado de citrato de sildenafilo y de un estímulo erótico visual puede ser eficaz para actuar como inducción farmacológica no invasiva (11). Además, esto puede servir para 
augurar la eficacia terapéutica de los inhibidores de la PDE5. Los parámetros utilizados para la evaluación vascular son los diámetros de las arterias cavernosas, la velocidad arterial sistólica máxima o pico, la velocidad arterial del final de la diástole, el tiempo de elevación sistólica, el tiempo de aceleración de las arterias cavernosas y el índice de resistencia vascular. La velocidad sistólica pico inferior a $25 \mathrm{~cm} / \mathrm{sg}$ después de la inyección intracavernosa y la estimulación sexual tiene una sensibilidad del $100 \%$ y una especificidad del $95 \%$ para seleccionar pacientes con arteriografía peneana anormal porque refleja una insuficiencia grave de las arterias cavernosas. Una velocidad sistólica pico constantemente mayor de $35 \mathrm{~cm} / \mathrm{sg}$ se asocia con una arteriografía normal y define un flujo de entrada normal en las arterias cavernosas. La ecografía doppler color evalúa también con precisión la disfunción eréctil por fracaso venooclusivo y debe realizarse antes de la cavernosografía y cavernosometría. Si el resultado del estudio ecográfico es normal no hay necesidad de continuar el examen vascular. Si el resultado es anormal, se debe realizar una arteriografía y cavernosografía/ cavernosometría de infusión dinámica únicamente a los pacientes que se considera potencialmente tributarios de cirugía vascular reconstructiva (12).

\section{Arteriografía selectiva de pene}

Hasta 1980, la arteriografía pudenda se consideraba como la prueba gold standard en el diagnóstico de la impotencia arteriogénica. En la actualidad, menos usada, sigue siendo la prueba diagnóstica más cruenta para detectar la $D E$ vasculogénica. Se realiza mediante la inyección intracavernosa de un agente vasodilatador (PGE 1), seguido de la canalización selectiva de la arteria pudenda interna y la inyección de contraste radiológico. Después se evalúan la anatomía y el aspecto radiológico de las arterias ilíacas, pudendas internas y peneanas (13).

La mejor indicación corresponde al paciente joven, menor de 50 años, con disfunción eréctil secundaria a lesión arterial traumática. En estos casos sumamente seleccionados es imprescindible un mapa vascular detallado de la anatomía arterial. Se busca un lugar aislado de oclusión arterial con posibilidades de corrección quirúrgica para planificar la reconstrucción mediante técnicas de anastomosis vasculares microquirúrgicas. La angiografía pudenda no está indicada cuando es imposible la revascularización quirúrgica (14).

También es usada para la embolización arterial en casos de priapismo de alta flujo (15).

\section{Cavernosometría y cavernosografía farmacológicas}

Previamente a 1998, la cavernosometría y la cavernosografía eran usadas frecuentemente para el diagnóstico de la DE masculina (16). La cavernosometría farmacológica comprende la infusión de solución fisiológica y la monitorización simultánea de la presión intracavernosa para evaluar el sistema de flujo de salida del pene después de la inyección intracavernosa de 20 microgramos de PGE 1 . En pacientes con disfunción cóorporo-veno-oclusiva se aprecia la incapacidad de aumentar la presión intracavernosa hasta el nivel de la presión sistólica media con la infusión de solución fisiológica o una caída rápida de la presión intracavernosa después de suspendida la infusión (17).

La realización de una cavernosografía farmacológica se basa en la infusión de solución de contraste radiológico en los cuerpos cavernosos después de una erección inducida por vasodilatadores para visualizar el sitio de pérdida o fuga venosa, que puede localizarse a nivel del glande, cuerpo esponjoso, venas dorsales superficiales y venas cavernosas y crurales. En la mayoría de los pacientes se visualiza más de un lugar de fuga. Con la relajación completa del músculo liso se describe que la velocidad de flujo necesaria para mantener la erección en una presión intracavernosa superior a $100 \mathrm{mmHg}$ es inferior a 3$5 \mathrm{ml} / \mathrm{min}$, mientras que la caída de la presión en 30 segundos desde $150 \mathrm{mmHg}$ es inferior a $45 \mathrm{mmHg}$. Si la presión desciende a una velocidad mayor de 45 $\mathrm{mm} \mathrm{Hg}$ en 30 segundos, indica que existe disfunción veno-oclusiva. Para confirmar radiográficamente un escape de la sangre venosa, también es útil la cavernosografía, que consiste en la inyección directa de un medio de contraste isoosmótico en goteo, seguido de la toma de una radiografía simple de la pelvis. Estas técnicas están indicadas en pacientes jóvenes candidatos a cirugía vascular del pene, para corregir una fuga veno-oclusiva de origen congénito o postraumático, y a veces, en los hombres con enfermedad de Peyronie y poca rigidez antes de emprender la cirugía reconstructiva del pene (18).

\section{Exploraciones Complementarias en DE: Valoración neurológica}

En todas las evaluaciones de la DE, la historia clínica y la exploración física preceden y son básicas para la ulterior evaluación neurológica. En general, la DE neurógena no exige el empleo de pruebas especializadas. A menudo, el paciente en quien se sospecha una $D E$ neurógena habrá sido diagnosticado anteriormente de algún proceso neurológico agudo o crónico. Cuando aparece una DE 
en edad juvenil, con comienzo agudo, y un resultado normal o muy bueno de la ecografía doppler peneana después de drogas vaso activas o iPDE5, son datos compatibles con DE de etiología neurógena. La evaluación neurológica tiene como finalidad evaluar los centros periféricos, medulares y supraespinales y las vías somáticas y autónomas asociadas con los tres tipos de erección y excitación sexual (19). Las pruebas neurológicas que pueden aplicarse son la velocidad de conducción nerviosa, la biotesiometría, la electromiografía (EMG) bulbocavernosa y la EMG de los cuerpos cavernosos. En un estudio reciente, se ha encontrado una estrecha relación entre los resultados anormales de las pruebas de sensibilidad térmica del pene y el diagnóstico clínico de DE neurogena (20). A pesar de las múltiples exploraciones neurológicas descritas, en escasas ocasiones el resultado de las mismas modifica la decisión terapéutica (1).

\section{Exploraciones Complementarias en DE: Registro de erección nocturna}

Este método es útil para individuos seleccionados. En su forma clásica la evaluación de la tumescencia peneana nocturna se basa en el empleo de dispositivos de monitorización nocturna que miden la cantidad de episodios eréctiles, la tumescencia (cambio circunferencial de calibres sin cambios de tensión), la rigidez peneana máxima y la duración de las erecciones nocturnas. Un hombre con función eréctil normal tiene de 4 a 6 episodios involuntarios de erecciones nocturnas durante el ciclo de sueño de 6 a 8 horas. Estas erecciones ocurren sobre todo durante la fase REM del sueño (rapid eye movement). En 1985 se introdujo el RigiScan $\Re_{\text {, que fue el primer }}$ dispositivo que permitió el registro automático y portátil de la tumescencia y la rigidez peneana nocturnas. El dispositivo combina la monitorización de la rigidez radial, la tumescencia, la cantidad y la duración de los eventos eréctiles con la conveniencia de un sistema portátil que se puede utilizar en el domicilio del paciente. Consiste básicamente en una unidad de registro consistente en dos anillos; uno ubicado en la base del pene y otro en el surco coronal que registran la circunferencia del pene y la rigidez radial en la base y la punta del pene. La cantidad de erecciones que se considera normal es de tres a seis por sesión de ocho horas, con una duración media de 10 a 15 minutos cada una (21). De aparición más reciente para la evaluación de la tumescencia peneana nocturna es el dispositivo NEVA®, que utiliza electrobioimpedancia para evaluar los cambios volumétricos en el pene durante las erecciones nocturnas (22).

Las principales ventajas de las pruebas de tumescencia peneana nocturna son la capacidad de evaluar la influencia psicológica en el inicio y mantenimiento de las erecciones y su capacidad de detectar anomalías relacionadas con el sueño. La presencia documentada de una erección completa indica que el eje neurovascular se encuentra funcionalmente intacto y que la causa de la disfunción eréctil muy probablemente sea psicógena.

Según Heaton y Morales las indicaciones para la valoración de la tumescencia y rigidez peneana nocturna son las siguientes: sospecha de trastorno del sueño, falta de respuesta al tratamiento, causa poco clara de disfunción eréctil, tratamiento quirúrgico planificado, caso legalmente difícil y sospecha de causa psicógena (23).

\section{CONCLUSIONES}

Las pruebas complementarias para una mejor compresión de la etiología de la disfunción eréctil pueden ser opcionales en ocasiones, y deben estar disponibles si el paciente desea profundizar en el diagnóstico etiológico de su enfermedad. Estas pruebas incluyen determinaciones analíticas en sangre, el registro de la tumescencia peneana nocturna, la ecografía doppler peneana, la cavernosometría, la cavernosografía, los test neurológicos y la inyección intracavernosa de prostaglandina $\mathrm{El}$, que se realiza generalmente, en la propia consulta. Todas a algunas de estas pruebas deben ser brindadas al paciente por parte del médico. La controversia existe en cuanto a la utilidad, valor relativo y las indicaciones de las mismas. Por norma general, y de manera evidente, estos test están dirigidos y realizados por urólogos quienes establecerán las indicaciones para pacientes específicos.

\section{BIBLIOGRAFIA y LECTURAS RECOMENDADAS ( ${ }^{*}$ lectura de interés $y^{* *}$ lectura fundamental)}

*1. Rosen R, Hatzichrstou D, Broderick G, et al. Clinical evaluation and symptom scales: sexual dysfunction assessment in men. En: Lue TF, Basson R, Rosen R, et al, editors. Sexual medicine: sexual dysfunctions in men and women, edition 2004. Paris: Health Publications; 2004. P.175-206

*2. Morales A, Heaton JP. Hormonal erectile dysfunction. Evaluation and management. Urol Clin North Am 2001;28(2):279-88.

**3. Lue TF, Giuliano F, Montorsi F, Rosen RC, Andersson KE, Althof S, Christ G, Hatzichristou D, Hirsch M, Kimoto Y, Lewis R, McKenna K, Ma- 
cMahon C, Morales A, Mulcahy J, Padma-Nathan H, Pryor J, de Tejada IS, Shabsigh R, Wagner G. Summary of the recommendations on sexual dysfunctions in men. J Sex Med 2004;1(1):6-23.

*4. Morales A, Buvat J, Gooren LJ, et al. Endocrine aspects of sexual dysfunction in men. J Sex Med. 2004;1(1):69-81.

5. Nehra A. Treatment of endocrinologic male sexual dysfunction. Mayo Clin Proc. 2000;75 Suppl:S40-5.

*6. Wespes E. The ageing penis. World J Urol. 2002;20(1):36-9.

*7. Shabsigh R. Testosterone therapy in erectile dysfunction and hypogonadism. J Sex Med. 2005;2(6):785-92.

8. Miner MM, Seftel AD. Testosterone and ageing: what have we learned since the Institute of Medicine report and what lies ahead? Int J Clin Pract. 2007;61(4):622-32.

9. Russell S, Nehra A. The physiology of erectile dysfunction. Herz 2003;28(4):277-83.

**10. Lobo JR, Nehra A. Clinical evaluation of erectile dysfunction in the era of PDE-5 inhibitors. Urol Clin North Am. 2005;32(4):447-55.

11. Speel TG, Bleumer I, Diemont WL, et al. The value of sildenafil as a mode of stimulation in pharmacopenile duplex ultrasonography. Int J Impot Res 2001;13(4):189-91.

12. Montorsi F, Bergamaschi F, Guazzoni G, et al. Velocity and flow volume gradients along the cavernosal artery: a duplex and color Doppler sonography study. Eur Urol. 1993;23(3):357-60.

13. Wahl SI, Rubin MB, Bakal CW. Radiologic evaluation of penile arterial anatomy in arteriogenic impotence. Int J Impot Res. 1997;9(2):93-7.

*14. Babaei AR, Safarinejad MR, Kolahi AA. Penile revascularization for erectile dysfunction: a systematic review and meta-analysis of effectiveness and complications. Urol J. 2009;6(1):1-7.

15. Ciampalini S, Savoca G, Buttazzi L, et al. Highflow priapism: treatment and long-term followup. Urology. 2002 Jan;59(1):110-3.

16. Stief CG, Diederichs W, Benard F, et al. The diagnosis of venogenic impotence: dynamic or pharmacologic cavernosometry? J Urol. 1988 Dec;140(6):1561-3.

17. Gamé X, Berrebi A, Lostes A, et al. Comparison of cavernometry and duplex ultrasound in the diagnosis of veno-occlusive erectile dysfunction. Prog Urol. 2006;16(4):470-3.

18. Vardi Y, Glina S, Mulhall JP, Menchini F, Munarriz R. Cavernosometry: is it a dinosaur?J Sex Med. 2008;5(4):760-4.

*19. Valles Antuña C, Fernández Gómez JM, Escaf S, et al. Etiología neurológica de la disfunción eréctil. Arch Esp Urol. 2008;61(3):403-11.

20. Lefaucheur KP, Yiou R, Colombel M et al. Relationship between penile termal sensory threshold-measurement and electrophysiologic test to assesss neurogenic impotence. Urology 2001;57(2):306-9.

21. Levine LA, Lenting EL. Use of nocturnal penile tumescence and rigidity in the evaluation of male erectile dysfunction. Urol Clin North Am. 1995;22(4):775-88.

22. Knoll LD, Abrams JH. Nocturnal electrobioimpedance volumetric assessment of patients with erectile dysfunction. Urol, 1999; 53(6):1200-4.

*23. Morales A, Condra M, Heaton JP, Johnston B, Fenemore J. Diurnal penile tumescence recording in the etiological diagnosis of erectile dysfunction. $\mathbf{J}$ Urol, 1994; 152(4):1111-4. 